Original Research - Short Report

\title{
Genomic convergence of locus-based GWAS meta-analysis identifies DDX11 as a novel Systemic Lupus Erythematosus gene
}

Mohammad Saeed ${ }^{1}$, Alejandro Ibáñez-Costa ${ }^{2}$, Alejandra María Patiño-Trives ${ }^{2}$, Eduardo Collantes Estevez $^{2}$, María Ángeles Aguirre², Chary López-Pedrera ${ }^{2}$

${ }^{1}$ ImmunoCure, Karachi, Pakistan

${ }^{2}$ Rheumatology Service, IMIBIC/Reina Sofia Hospital, University of Cordoba, Cordoba, Spain

Running Title: DDX11 and SLE

Title character count: 115

Abstract word count: 180

Text word count: 1,536

Conflict of Interest: None

Funding: As in Acknowledgements

Author ORCID ID (in authorline order): 0000-0002-5276-5940, 0000-0003-4649-0095, 0000-0001-

9198-0347, 0000-0002-7647-6289, 0000-0002-5653-4293, 0000-0003-2067-4603

Correspondence:

Dr. Mohammad Saeed, M.D.

ImmunoCure

Suite 116, $\mathbf{1}^{\text {st }}$ Floor, The Plaza, 2-Talwar, Clifton

Karachi, Pakistan

Email: msaeed@immunocure.pk

\&

Dr. Chary López-Pedrera, MSc, PhD.,

Maimonides Institute for Research in Biomedicine of Cordoba

Avda. Menéndez Pidals/n,

Córdoba E-14004, Spain

Email: rosario.lopez.exts@juntadeandalucia.es 


\title{
Summary Statement
}

More than 100 genes for SLE have been identified but they explain only $\sim 15 \%$ of heritability. GWAS are challenged by risk genes of modest effect. Using locus-based GWAS mapping and multiple gene expression replications, DDX11 was identified as a novel SLE gene. DDX11, repressed in SLE, may be used as a clinical diagnostic tool.

\begin{abstract}
Genome-wide association studies (GWAS) of systemic lupus erythematosus (SLE) explain only $\sim 15 \%$ of genetic risk, indicating genes of modest effect remain to be discovered. Association clustering methods such as OASIS are more apt at identifying modest genetic effects. 410 genes were mapped to previously identified OASIS GWAS SLE loci and investigated for expression in SLE GEO datasets. GSE50395 dataset from Cordoba was used for validation. Blood eQTL for significant SNPs in SLE loci and STRING for functional pathways of differentially expressed genes was used. Confirmatory qPCR on monocytes of 12 SLE patients and controls was performed. We identified 55 genes that were differentially expressed in at least 2 SLE GEO datasets with all probes directionally aligned. $D D X 11$ was downregulated in both $\mathrm{GEO}(\mathrm{P}=3.60 \mathrm{E}-02)$ and Cordoba $(P=8.02 \mathrm{E}-03)$ datasets and confirmed by qPCR $(P=0.001)$. The most significant SNP, rs3741869 $(P=3.2 \mathrm{E}-05)$ in OASIS locus $12 \mathrm{p} 11.21$, containing $D D X 11$, was a cis-eQTL regulating DDX11 expression $(\mathrm{P}=8.62 \mathrm{E}-05) . D D X 11$ interacted with multiple genes including STAT1/STAT4. Genomic convergence with OASIS and multiple expression datasets identifies novel genes. DDX11, RNA helicase involved in genome stability, is repressed in SLE.
\end{abstract}

\section{Key words: lupus; DDX11; OASIS.}




\section{Introduction}

Systemic lupus erythematosus (SLE) is a diverse complex disorder, manifesting more like syndromes than a singular disease (Saeed. 2017a). SLE could therefore be thought of as a mixture of multiple resembling phenotypes, each a result of a separate mutation, pooled in genome-wide association study (GWAS) cohorts (Saeed. 2017b). Finding a particular gene then depends on the enrichment of a causal mutation carrying haplotype in the study sample.

Genotyping a multitude of single-nucleotide polymorphisms (SNPs) in GWAS leads to the multiple testing problem, preventing the true association signal from being distinguished from random noise. Despite the identification of large number of genes in multiple comprehensive SLE GWAS and candidate gene studies, they together explain only $15 \%$ of SLE heritability (Bentham et al. 2015; Morris et al., 2016).

OASIS is a linkage disequilibrium (LD) clustering method that can be used to mine existing complex disease GWAS datasets for new genes (Saeed, 2017b, Saeed, 2018). OASIS provides an alternative to increasing sample size for GWAS by composite analysis unifying two aspects of the LD phenomenon - strength of association and number of surrounding significant SNPs. A genomic convergence approach (Liang et al., 2009) mapping genetic association signals on expression data and other biological studies can then be used for verification of particular genes.

Previously, OASIS analysis of two dbGAP GWAS datasets (6077 subjects; 0.75 million SNPs) identified thirty SLE associated loci (Saeed, 2017b). In the present study, 410 genes were mapped to these loci and investigated for gene expression in three SLE GEO datasets. A fourth expression dataset from Cordoba, Spain was used for validation. This study showed that DDX11, a RNA helicase involved in genome stability, is repressed in SLE. Thus, a genomic convergence approach with OASIS, and expression studies on multiple datasets can help identify novel SLE genes. 


\section{Results}

\section{Genomic Convergence}

SNIPPER located 410 genes (Table S1) in 30 SLE loci identified by OASIS (Saeed 2017b) and these were tested in 3 GEO expression datasets. This search identified over 1000 expression probe sets in GEO datasets GSE30153, GSE13887 and more than 500 probe sets in GEO dataset GSE10325 for the reference gene list. Significant T-tests for these probe tests identified, 103 probes in dataset 4193 (GSE30153), 141 probes in dataset 4719 (GSE13887) and 66 probes in dataset 4185 (GSE10325). Additional 215 probes were found to be significant in GSE10325: Tcells, B-cells and Monocytes datasets. Hence, a total of 525 statistically significant probe sets in six (3+3) GEO datasets were finalized. This composite probe list was matched against the SNIPPER reference gene list of 410 genes, to remove those that the GEO query included, however, were not found in the OASIS SLE loci. Hence, 187 unique genes in the 6 GEO datasets were identified that had significant change in expression and were located in the 30 SLE loci (Table S2).

\section{Expression Validation}

Of the 187 candidate genes, 55 genes were found to be differentially expressed in at least 2 GEO datasets with all probes directionally aligned (Table S3). Four Genes crossed the false discovery rate (FDR) viz STAT1, IFIH1, NRF1 and TTC9 (Table 1). DDX11, located in OASIS locus 19 on chromosome 12, was downregulated, however it did not cross the FDR for expression. In the Cordoba expression dataset, GSE50395, 518 genes were found to have differential expression. Of these 10 genes were found in the reference list of 410 genes in OASIS SLE loci (Table 2). BARHL1 had the most significant expression change $\left(P=8.9 \times 10^{-5}\right)$ and $D D X 11$ had the second highest expression change $\left(P=8 \times 10^{-3}\right)$.

\section{$e Q T L$ analysis and $q P C R$}

In 6 of 10 genes identified in the Cordoba dataset (GSE50395) that mapped onto OASIS loci, eQTLs were detected. The most significant SNP rs3741869 (GWAS P=3.2E-05) in OASIS locus 19 (chromosome 12p11.21), containing the gene DDX11, was found to be a cis-eQTL regulating the expression of DDX11 ( $\mathrm{P}=8.62 \mathrm{E}-05)$. The other GWAS 
significant SNPs modulated expression of genes not significant in GEO or Cordoba datasets (Table S4). Hence, DDX11 was the only gene that could be functionally verified in this study by genomic convergence.

Expression of DDX11 was confirmed with qPCR in monocytes isolated from 12 SLE patients and 12 healthy donors. As shown in Figure 2, DDX11 was significantly downregulated in SLE patients $(P=0.0010)$. We adjusted the data as logarithms, however despite any normalization technique the results remained the same.

\section{Protein network analysis}

Protein network analysis using STRING of 187 (55+132) genes with significant differential expression in GEO datasets showed a complex interaction network. DDX11 interacted indirectly with several genes that were found to be significant in OASIS loci and GEO datasets. DDX11 interaction with STAT1/STAT4 was mediated via RIF1, RBM25 and then BAZ1A (Figure S1a). Interaction analysis of 55 genes having significant differential expression in at least 2 GEO datasets and with their expression directionally aligned, showed that DDX11 directly interacted with MED6 (interaction score 0.31) (Figure S1b). Interestingly, the remaining 132 genes included several known SLE genes such as IRF5, BLK, TNIPI and CD44, however these genes were either significant in a single GEO dataset only or did not have a uniform up-/ down-regulation for the significant probes.

\section{Discussion}

This study employed a genetic and functional analysis using OASIS, a locus-based test, and multiple gene expression datasets to identify novel SLE genes. The most important finding of this study was the identification of DDX11 which was significantly downregulated in both the GEO (P=3.60E-02) and the Cordoba (P=8.02E-03) SLE expression datasets. $D D X 11$ downregulation in SLE was confirmed $(P=0.001)$ using qPCR on monocytes. The most significant SNP rs3741869 in OASIS locus 19 containing the gene DDX11, was found to be a cis-eQTL regulating the expression of $D D X 11$ ( $\mathrm{P}=8.62 \mathrm{E}-05) . D D X 11$ interacted with multiple known SLE genes including STAT1/STAT4, identified using genomic convergence of OASIS loci and expression analysis. 
DEAD/H (Asp-Glu-Ala-Asp/His) box helicase, DDX11 is a RNA helicase thought to be involved in genome stability. It is located at the 12p11.21 locus that associated with SLE in two GWAS. Moreover, DDX11 was found to be downregulated in SLE in multiple expression datasets and this finding was confirmed with qPCR in SLE monocytes. These findings confirm $D D X 11$, a DNA helicase, as a novel SLE gene. $D D X 11$ mutations cause the Warsaw Breakage Syndrome (WABS), an autosomal recessive cohesinopathy with the clinical triad of severe congenital microcephaly, growth restriction, and sensorineural hearing loss (Eppley et al., 2017). WABS shows features of genome instability similar to Fanconi anemia (FA). Several genes are involved in the FA pathway which is critical to DNA repair of interstrand crosslinks (ICL). DDX11 functions as a FA pathway backup and its deficiency leads to impaired ICL repair (Abe et al., 2018). Moreover, DDX11 plays an important role in B-cell immunoglobulin (Ig) diversification (Abe et al., 2018) which makes it an even more interesting candidate gene for SLE. Inhibition of $D D X 11$ expression led to extensive apoptosis in melanoma cells (Bhattacharya et al. 2012). Previously, another melanoma associated gene, Melanoma differentiation antigen 5 (MDA5), has been shown to be important in SLE (Saeed, 2017a).

Though DDX11 was the only gene the present study could confirm as significant in SLE, we identified several other candidate genes as well. These include $N R F 1, T T C 9$ and BARHL1, which need additional data for verification. Hence, replication based genomic convergence approach with OASIS, and expression studies can help identify novel SLE genes. Measuring DDX11 expression by qPCR in monocytes from SLE patients as described here, can potentially be used as a clinically useful predictive marker for SLE. This may allow early recognition and treatment of SLE possible and may significantly impact prevention of SLE complications.

\section{Methods}

\section{Datasets}

GWAS datasets were obtained online from dbGAP repository. Meta-analysis of two SLE datasets, phs000202 (Harley et al 2008) and phs000122 (Hom et al. 2008), was conducted using OASIS (Saeed, 2017b). The dataset phs000202 consisted of 706 SLE females and 353 controls and was used for screening (Harley et al 2008), while phs000122, comprising of 1435 SLE cases and 3583 controls genotyped for 500K single nucleotide polymorphisms 
(SNPs), was used as the replication dataset (Hom et al. 2008). SNIPPER was used to identify genes in OASIS loci. This list of reference genes was tested in 3 GEO datasets (GSE30153, GSE13887, GSE10325) for expression. Two datasets (GSE30153, GSE13887) had SLE cases and healthy controls whereas GSE10325 had three cellular fractions (T, B cells and Monocytes) resulting in 3 additional datasets (GSE10325: T, B, M). A fourth geneexpression dataset, GSE50395, from Cordoba, Spain was used for validation (Perez-Sanchez et al, 2015). However, the expression analysis comparing SLE with healthy controls is new. Figure 1 details the methodology for gene discovery.

eQTL Analysis and $q P C R$

Significant SNPs in loci harboring differentially expressed genes in SLE were tested using Blood eQTL Browser (expression quantitative trait loci) (Westra et al., 2013). We confirmed the expression of DDX11 using qPCR on monocytes of 12 healthy donors and 12 SLE patients from Cordoba, Spain. Specific primers for DDX11 were previously reported (Hormaechea-Agulla et al, 2017).

\section{Protein network analysis}

All genes with significant and consistent directional change in expression in the GEO datasets were tested for protein-protein interactions using STRING (Szklarczyk et al., 2015). Gene pairs that were either co-expressed or involved in experimentally validated interactions with at least a low score $(0.15)$ were evaluated.

Acknowledgements. The authors thank all patients and healthy subjects for participation in the study. This work was accepted as a Poster in the $12^{\text {th }}$ European Lupus Meeting. Due to the COVID19 global outbreak the Meeting was canceled and this work could not be presented, however, the Abstract was recently published http://dx.doi.org/10.1136/lupus-2020-eurolupus.136

Contributors All authors were involved in drafting the article or revising it critically for important intellectual content, and all authors approved the final version to be published. 
Funding. This study was supported by grants from the Instituto de Salud Carlos III (PI18/00837), cofinanciado por el Fondo Europeo de Desarrollo Regional de la Unión Europea “Una manera de hacer Europa," Spain, and the Spanish Inflammatory and Rheumatic Diseases Network (RIER), Instituto de Salud Carlos III (RD16/0012/0015).

C.L-P was supported by a contract from the Spanish Junta de Andalucía ("Nicolas Monardes” program).

Competing interests: None declared.

Patient consent for publication: Not required. The study was approved by the Ethical Research Committee of Hospital Universitario Renia Sofia.

Provenance and peer review: Not commissioned; externally peer reviewed.

Data availability statement: All data relevant to the study are included in the article or uploaded as supplementary information. All datasets are in publically available repositories with their accession numbers in the manuscript.

\section{Author ORCID ID (in authorline order):}

Mohammad Saeed ${ }^{\mathbf{1}, 0000-0002-5276-5940}$

Alejandro Ibáñez-Costa ${ }^{2}$, 0000-0003-4649-0095

Alejandra María Patiño-Trives ${ }^{2}, 0000-0001-9198-0347$

Eduardo Collantes Estevez ${ }^{2}$, 0000-0002-7647-6289

María Ángeles Aguirre², 0000-0002-5653-4293

Chary López-Pedrera ${ }^{2}$, 0000-0003-2067-4603

\section{Web Resources}

OASIS software (Python 2.7.9 code): https://github.com/dr-saeed/OASIS/blob/master/OASIS.py

SNIPPER: http://csg.sph.umich.edu/boehnke/snipper/

Blood eQTL Browser: https://genenetwork.nl/bloodeqtlbrowser/

STRING: $\underline{\text { http://string-db.org }}$ 


\section{References}

Abe T, Ooka M, Kawasumi R, Miyata K, Takata M, Hirota K, Branzei D. (2018). Warsaw breakage syndrome DDX11 helicase acts jointly with RAD17 in the repair of bulky lesions and replication through abasic sites. Proc Natl Acad Sci U S A. 115(33):8412-8417. PMID: 30061412.

Bentham J, Morris DL, Cunninghame Graham DS, Pinder CL, Tombleson P, Behrens TW, Martín J, et al. (2015) Genetic association analyses implicate aberrant regulation of innate and adaptive immunity genes in the pathogenesis of systemic lupus erythematosus. Nat Genet. 47(12):1457-64. PMID: 26502338.

Bhattacharya C, Wang X, Becker D. (2012). The DEAD/DEAH box helicase, DDX11, is essential for the survival of advanced melanomas. Mol Cancer. 11:82. PMID: 23116066.

Eppley S, Hopkin RJ, Mendelsohn B, Slavotinek AM. (2017). Clinical Report: Warsaw Breakage Syndrome with small radii and fibulae. Am J Med Genet A. 173(11):3075-3081. PMID: 28960803.

Hom G, Graham RR, Modrek B, Taylor KE, Ortmann W, Garnier S, Lee AT, et al. (2008) Association of systemic lupus erythematosus with C8orf13-BLK and ITGAM-ITGAX. N Engl J Med. 358(9):900-9. PMID: 18204098.

Hormaechea-Agulla D, Gahete MD, Jiménez-Vacas JM, Gómez-Gómez E, Ibáñez-Costa A, L-López F, RiveroCortés E, Sarmento-Cabral A, et al. (2017). The oncogenic role of the In1-ghrelin splicing variant in prostate cancer aggressiveness. Mol Cancer. 16(1):146. PubMed PMID: 28851363.

International Consortium for Systemic Lupus Erythematosus Genetics (SLEGEN), Harley JB, Alarcón-Riquelme ME, Criswell LA, Jacob CO, Kimberly RP, Moser KL, Tsao BP, et al. (2008) Genome-wide association scan in women with systemic lupus erythematosus identifies susceptibility variants in ITGAM, PXK, KIAA1542 and other loci. Nat Genet. 40(2):204-10. PMID: 18204446.

Liang X, Slifer M, Martin ER, Schnetz-Boutaud N, Bartlett J, Anderson B, Züchner S, Gwirtsman H, Gilbert JR, Pericak-Vance MA, Haines JL. (2009). Genomic convergence to identify candidate genes for Alzheimer disease on chromosome 10. Hum Mutat. 30(3):463-71. PMID: 19241460. 
Morris DL, Sheng Y, Zhang Y, Wang YF, Zhu Z, Tombleson P, Chen L, et al. (2016). Genome-wide association meta-analysis in Chinese and European individuals identifies ten new loci associated with systemic lupus erythematosus. Nat Genet. 48(8):940-946. PMID: 27399966.

Perez-Sanchez C, Barbarroja N, Messineo S, Ruiz-Limon P, Rodriguez-Ariza A, Jimenez-Gomez Y, Khamashta MA, Collantes-Estevez E, et al. (2014). Gene profiling reveals specific molecular pathways in the pathogenesis of atherosclerosis and cardiovascular disease in antiphospholipid syndrome, systemic lupus erythematosus and antiphospholipid syndrome with lupus. Ann Rheum Dis. 74(7):1441-9. PMID: 24618261.

Saeed M. (2017a). Lupus pathobiology based on genomics. Immunogenetics. 69(1):1-12. PMID: 27933432.

Saeed M. (2017b). Novel linkage disequilibrium clustering algorithm identifies new lupus genes on meta-analysis of GWAS datasets. Immunogenetics. 2017;69(5):295-302. PMID: 28246883.

Saeed M. (2018). Genomic convergence of locus-based GWAS meta-analysis identifies AXIN1 as a novel Parkinson's gene. Immunogenetics. 70(9):563-570. PMID: 29923028.

Szklarczyk D, Franceschini A, Wyder S, Forslund K, Heller D, Huerta-Cepas J, Simonovic M, et al. (2015). STRING v10: protein-protein interaction networks, integrated over the tree of life. Nucleic Acids Res. 43(Database issue):D447-52. PMID: 25352553.

Westra HJ, Peters MJ, Esko T, Yaghootkar H, Schurmann C, Kettunen J, Christiansen MW, et al. (2013). Systematic identification of trans eQTLs as putative drivers of known disease associations. Nat Genet. 45(10):12381243. PMID: 24013639. 


\section{Legends}

\section{Figure 1. Methodology for gene discovery and validation}

Genomic convergence methodology employed in this study. Two GWAS (Db1 and Db2) were analyzed using OASIS locus-based testing, which identified 30 overlapping SLE loci. SNIPPER was used to identify 410 genes in these 30 loci and this reference gene list was used for gene expression analysis in 3 SLE GEO datasets and 1 dataset from Cordoba, Spain. DDX11 was the only gene identified across all expression datasets with consistently significant expression change in SLE.

\section{Figure 2. DDX11 qPCR of monocytes from SLE and healthy donors}

qPCR confirmation of DDX11 expression in monocytes isolated from 12 SLE patients and 12 healthy donors. DDX11 is downregulated in SLE patients. Data was adjusted as logarithms. Logarithmic Normalized DDX11/GADPH: unpaired t-test, $\mathrm{P}=0.0010$. DDX11/GAPDH: Mann Whitney test, $\mathrm{P}=0.0096$.

\section{Figure S1. DDX11 Protein network analysis}

STRING protein network analysis of 187 unique genes (S1a) that had significant change in expression in 6 GEO datasets and were located in the 30 SLE loci. STRING analysis of 55 genes (S1b) having significant differential expression in at least 2 GEO datasets and with their expression directionally aligned. Minimum interaction score of 0.15 was chosen for either Co-expression or Experimental interaction.

Table 1. Top SLE candidate genes by genetic association and gene expression validation

Of 55 genes that showed statistically significant and consistent expression change in at least 2 GEO datasets, 4 crossed the FDR. STAT4 and IFIH1 are known SLE genes, however NRFI and TTC9 need further data for validation. DDXI1 did not cross the FDR. 


\section{Table 2. Genomic Convergence of 410 reference genes in Cordoba Expression Dataset}

Ten of 518 genes with expression change in the Cordoba dataset, were present in the reference gene list (410 genes). $D D X 11$ had the second highest expression change $\left(P=8 \times 10^{-3}\right)$.

Table S1. Reference list of genes in OASIS identified SLE loci

In 30 overlapping SLE loci identified by OASIS in two GWAS datasets, 410 genes were found using SNIPPER

Table S2. Genomic Convergence of the reference genes in GEO Expression Datasets

In 6 GEO datasets, 187 unique genes were identified that had significant change in expression and were located in the 30 SLE loci.

Table S3. Genes with significant and consistent expression change in at least 2 GEO datasets

Of the 187 candidate genes 55 were found to be differentially expressed in at least 2 GEO datasets with all probes directionally aligned.

Table S4. Expression quantitative trait locus (eQTL) analysis of significant genes in Cordoba validation dataset

eQTLs were found in 6 significantly expressed genes in the Cordoba dataset. DDX11 expression was modulated by the SNP rs3741869 that showed maximum significance in the DDX11 genomic locus (OASSI locus 19). The other eQTL SNPs modulated expression of genes not found to be significant in this study. Interestingly ITGAM/ITGAX, which are known SLE genes, were regulated by the SNPs rs11574637 and rs9888739, located in TRIM72 and functioned as cis-eQTLs. 
Figure 1. Methodology for gene discovery and validation

NRF1, TTC9 \& STAT1, IFIH1

Highly differentially expressed in multiple SLE datasets

DDX11 only gene out of 55 validated for expression in Cordoba dataset. It is down regulated in SLE.

\section{Db1}

706 SLE females / 353 controls. 258K SNPs

phs000202 (Harley et al. 2008)

[PMID: PMID: 18204446]
$\mathrm{Db2}$

1435 SLE cases / 3583 controls $500 \mathrm{~K}$ SNPs

phs000122 (Hom et al. 2008)

[PMID: 18204098].

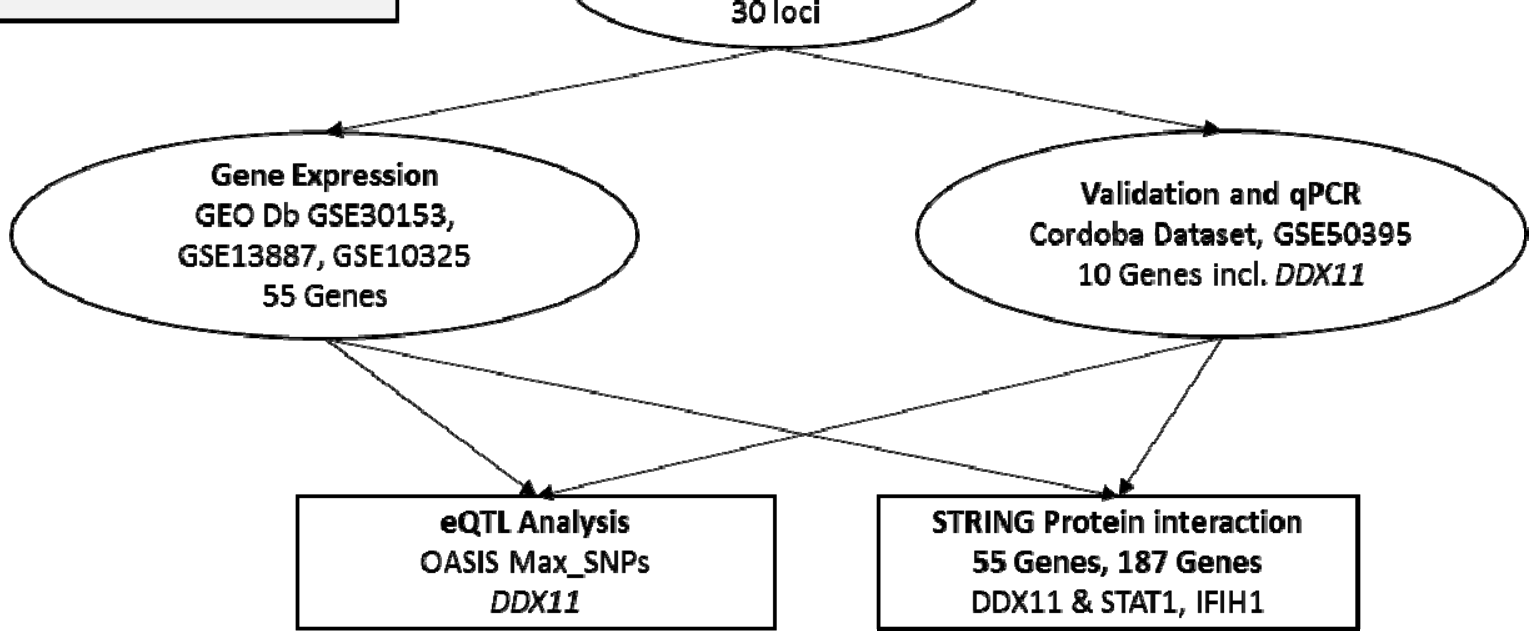


Figure 2. DDX11 qPCR of monocytes from SLE and healthy donors

\section{DDX11}

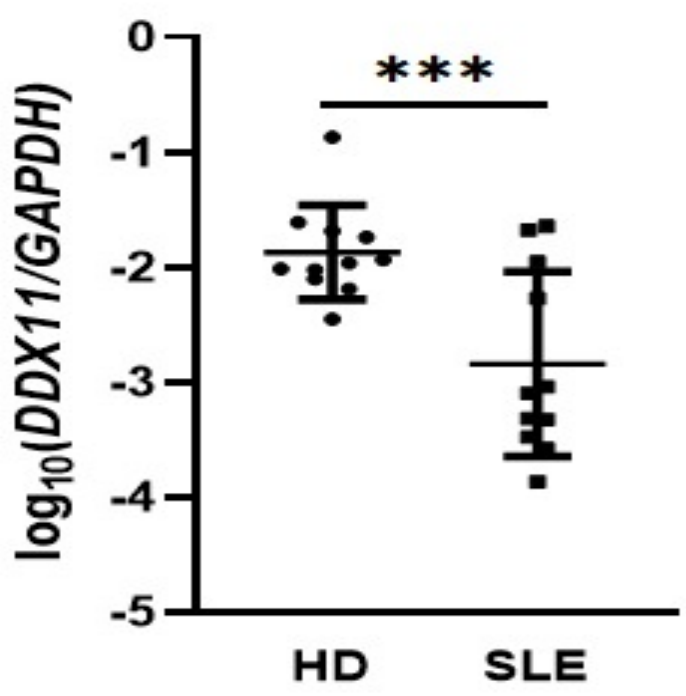


Table 1. Top SLE candidate genes by genetic association and gene expression validation

\begin{tabular}{|c|c|c|c|c|c|c|c|c|c|c|}
\hline \multicolumn{6}{|c|}{ Genetic Association } & \multicolumn{5}{|c|}{ Gene Expression } \\
\hline Gene & Chr: Position (bp) & Locus & $\begin{array}{c}\text { Quad } \\
(\text { Db1, Db2) }\end{array}$ & Max_SNP (Db1, Db2) & $\begin{array}{c}\text { Max_P } \\
(\text { Db1, Db2) }\end{array}$ & $\begin{array}{c}\text { Significant } \\
\text { Probes }\end{array}$ & T_Ave (P) & T-Min (P) & FDR (P) & $\begin{array}{c}\text { Ave_Fold } \\
\text { Change }\end{array}$ \\
\hline STAT1 & 2: $191838203 \ldots 192060779$ & 3 & $\mathrm{~A}, \mathrm{~B}$ & rs7574865, rs3771327 & $13.05,4.06$ & 26 & $4.64 \mathrm{E}-03$ & 7.75E-08 & $9.26 \mathrm{E}-04$ & 36.19 \\
\hline IFIH1 & 2: $161182794 \ldots 163311201$ & 2 & $\mathrm{C}, \mathrm{B}$ & rs6755940, rs13023380 & $3.12,4.16$ & 8 & $1.41 \mathrm{E}-02$ & 7.19E-05 & $9.43 \mathrm{E}-04$ & 25.02 \\
\hline$N R F 1$ & 7: $128393949 \ldots 132065378$ & 9 & $\mathrm{~B}, \mathrm{~B}$ & rs4728142, rs10488631 & $6.14,10.78$ & 6 & $3.00 \mathrm{E}-02$ & $7.98 \mathrm{E}-05$ & $9.62 \mathrm{E}-04$ & 0.67 \\
\hline TTC9 & 14: $69831036 \ldots 73507392$ & 25 & $\mathrm{C}, \mathrm{C}$ & rs7141009, rs2052219 & $2.73,3.09$ & 3 & $1.93 \mathrm{E}-02$ & $1.20 \mathrm{E}-04$ & $9.80 \mathrm{E}-04$ & 0.76 \\
\hline$D D X 11$ & 12: $30383902 \ldots 31288883$ & 19 & $\mathrm{C}, \mathrm{B}$ & rs10843682, rs3741869 & $1.89,4.50$ & 2 & $3.90 \mathrm{E}-02$ & $3.60 \mathrm{E}-02$ & $2.94 \mathrm{E}-03$ & 0.7 \\
\hline
\end{tabular}


Table 2. Genomic Convergence of 410 reference genes in Cordoba Expression Dataset

\begin{tabular}{|c|c|c|c|c|c|c|c|c|}
\hline Gene & FC Abs & P-value & Chr & $\begin{array}{l}\text { OASIS } \\
\text { Locus }\end{array}$ & Locus Position & Max_SNP (db1, db2) & $\begin{array}{c}\text { Max_-log(P) } \\
(\mathrm{db} 1, \mathbf{d b 2})\end{array}$ & Quadrant \\
\hline BARHLI & -2.66 & $8.85 \mathrm{E}-05$ & $9 q 34.13$ & 13 & 9: $135083870 . .135453438$ & rs11243676, rs1185995 & $5.58,4.97$ & $\mathrm{~B}, \mathrm{~B}$ \\
\hline DDX11 & -2.08 & $8.02 \mathrm{E}-03$ & $12 \mathrm{p} 11.21$ & 19 & 12: $30383902 . .31288883$ & rs 10843682, rs 3741869 & $1.89,4.50$ & $\mathrm{C}, \mathrm{B}$ \\
\hline TRIM72 & -2.30 & $1.79 \mathrm{E}-02$ & $16 \mathrm{p} 11.2$ & 27 & 16: $31193941 . .31527819$ & rs11574637, rs9888739 & $6.27,4.00$ & $\mathrm{~B}, \mathrm{~B}$ \\
\hline RBMS1 & -2.88 & 2.39E-02 & $2 q 24.2$ & 2 & 2: $161182794 . .163311201$ & rs6755940, rs13023380 & $3.12,4.16$ & $\mathrm{C}, \mathrm{B}$ \\
\hline CDKN1B & 2.47 & $2.76 \mathrm{E}-02$ & $12 \mathrm{p} 13.1$ & 18 & 12: $12221429 . .13759033$ & rs7980903, rs4764011 & $4.39,3.26$ & $\mathrm{~B}, \mathrm{C}$ \\
\hline$A D A M 21$ & -3.19 & $2.97 \mathrm{E}-02$ & $14 q 24.2$ & 25 & 14: $69831036 . .73507392$ & rs7141009, rs2052219 & $2.73,3.09$ & $\mathrm{C}, \mathrm{C}$ \\
\hline ZNF385D & -4.66 & $3.89 \mathrm{E}-02$ & $3 p 24.3$ & 5 & 3: $21523341 . .23226297$ & rs3860579, rs680930 & $3.85,3.06$ & $\mathrm{~B}, \mathrm{C}$ \\
\hline LOC100286922 & -2.25 & 4.01E-02 & $2 q 37.1$ & 4 & 2: $234514762 . .234825092$ & rs 17863787, rs4663968 & $3.17,2.61$ & $\mathrm{C}, \mathrm{C}$ \\
\hline LOH12CR2 & 2.13 & $4.34 \mathrm{E}-02$ & $12 \mathrm{p} 13.2$ & 18 & 12: $12221429 . .13759033$ & rs7980903, rs4764011 & $4.39,3.26$ & $\mathrm{~B}, \mathrm{C}$ \\
\hline ELF5 & -2.21 & $4.47 \mathrm{E}-02$ & $11 \mathrm{p} 13$ & 15 & 11: $34821947 . .35251317$ & rs519858, rs2785197 & $2.57,3.41$ & $\mathrm{C}, \mathrm{C}$ \\
\hline
\end{tabular}


Table S4. Expression quantitative trait locus (eQTL) analysis of significant genes in Cordoba validation dataset

\begin{tabular}{|c|c|c|c|c|c|c|c|c|}
\hline OASIS Locus & Gene & cis / trans & P-value & SNP & Probe & SNP Alleles & Z-score & Modulated Gene \\
\hline 13 & BARHL1 & trans & 3.21E-06 & rs11243676 & 5900274 & G>A & 4.66 & EDA \\
\hline 13 & BARHL1 & cis & 2.93E-06 & rs1185995 & 1340114 & G>A & 4.68 & TTF1 \\
\hline 18 & CDKN1B & cis & 1.37E-03 & rs7980903 & 2060273 & $\mathbf{T}>\mathbf{C}$ & -3.20 & BCL2L14 \\
\hline 18 & LOH12CR2 & cis & 1.37E-03 & rs7980903 & 2060273 & $\mathbf{T}>\mathbf{C}$ & -3.20 & BCL2L14 \\
\hline 19 & $D D X 11$ & cis & 8.62E-05 & rs3741869 & 4890750 & $\mathbf{G}>\mathbf{A}$ & 3.93 & $D D X 11$ \\
\hline 25 & $A D A M 21$ & cis & 1.10E-19 & rs7141009 & 4900164 & $\mathbf{T}>\mathbf{G}$ & 9.08 & - \\
\hline 25 & $A D A M 21$ & cis & 5.55E-14 & rs7141009 & 3170093 & $\mathbf{T}>\mathbf{G}$ & -7.52 & KIAA0247 \\
\hline 25 & $A D A M 21$ & cis & 4.31E-19 & rs2052219 & 940129 & G>A & -8.93 & WDR21A \\
\hline 27 & TRIM72 & trans & 1.10E-05 & rs11574637 & 4920242 & $\mathbf{T}>\mathbf{C}$ & -4.40 & - \\
\hline 27 & TRIM72 & cis & 9.91E-33 & rs11574637 & 4490500 & $\mathbf{T}>\mathbf{C}$ & 11.91 & ITGAX \\
\hline 27 & TRIM72 & cis & 2.18E-06 & rs11574637 & 1710070 & $\mathbf{T}>\mathbf{C}$ & 4.74 & AC093520.4,ITGAM \\
\hline 27 & TRIM72 & cis & 1.31E-22 & rs9888739 & 4490500 & $C>T$ & 9.79 & ITGAX \\
\hline 27 & TRIM72 & cis & $6.56 \mathrm{E}-06$ & rs9888739 & 1710070 & $C>T$ & 4.51 & AC093520.4,ITGAM \\
\hline 27 & TRIM72 & cis & 2.11E-03 & rs9888739 & 5220347 & $C>T$ & 3.07 & BCKDK \\
\hline
\end{tabular}




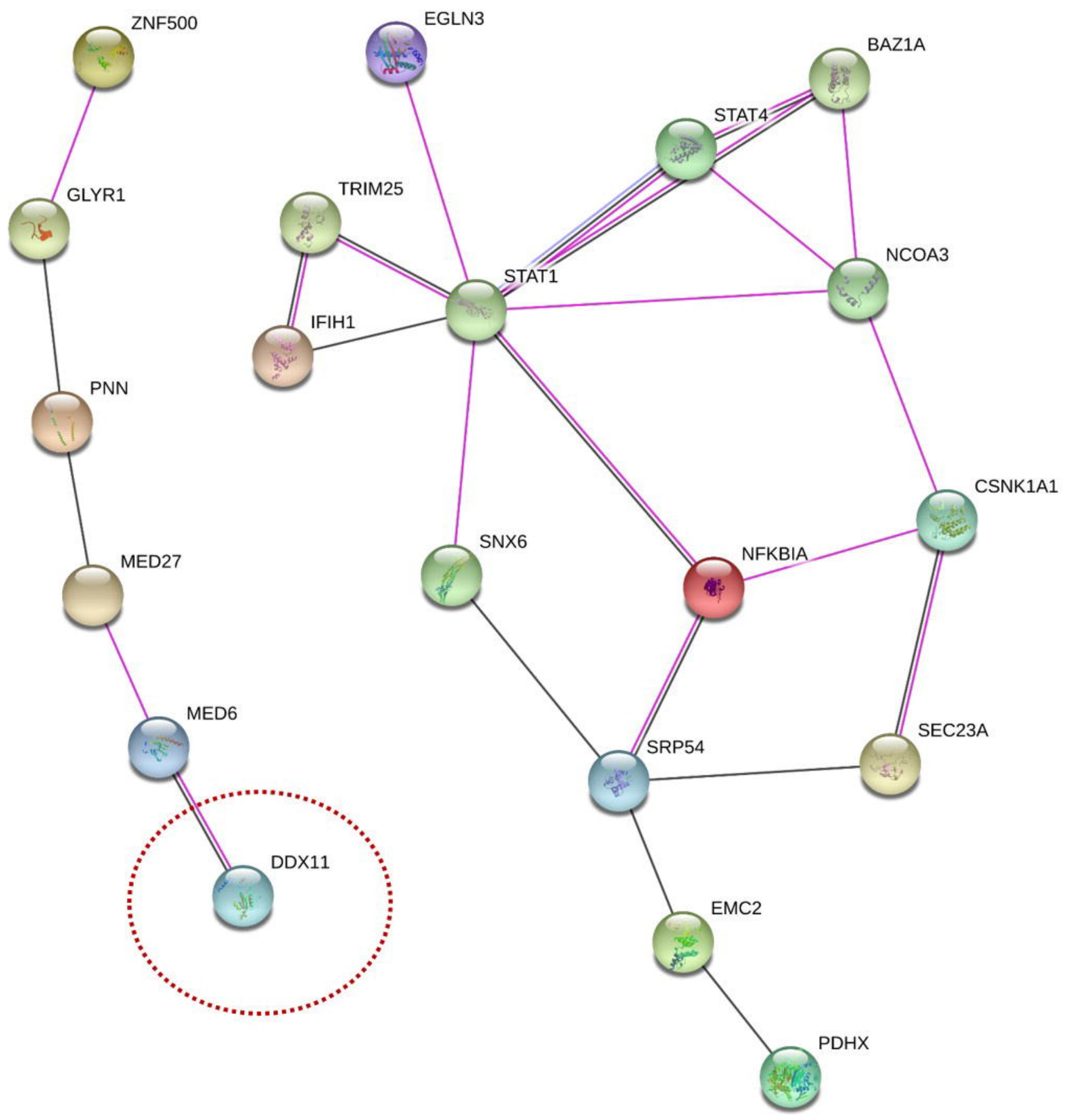

